\title{
Scoping the international impact from four independent national dermatology trials
}

Williams $\mathrm{HC}^{1}$, Rogers $\mathrm{NK}^{1}$, Chalmers $\mathrm{JR}^{1}$, Thomas $\mathrm{KS}^{1}$

${ }^{1}$ Centre of Evidence-Based Nottingham, Nottingham, UK

Corresponding author: Prof. Hywel C. Williams, hywel.williams@nottingham.ac.uk ORCID 0000-0002-56463093

Running header: Scoping the impact from four dermatology trials

Word count: 2,266 . One box. One supplementary table. No figures. 23 references. Submitted as an Original Article.

\section{Abstract}

Background: Research impact describes whether and how research results in wider benefits to society beyond academic publication. Little is known about translation of clinical trial research into dermatological practice .

Objectives: We scoped international impact from four independently funded clinical trials published by our group over the last 10 years.

Methods: Scoping survey of 35 international colleagues from 22 countries followed by a narrative summary of emergent themes.

Results: All kindly responded to the survey. At least 20 emergent themes were identified and broadly included: (i) interest and enthusiasm in the concept of trying to document clinical trial impact (ii) direct impacts such as adoption of the drug as tested and recommended from the trial results including more confidence using the drug in slightly different ways for the same condition, (iii) the finding that trial impact was dependent on factors such as drug availability and country specific disease patterns and (iv) the educational value of good trial design for journal club discussions and improving future clinical trial designs in dermatology. Our survey suggests that uptake into clinical practice was surprisingly rapid and widespread.

Conclusions: Clinical trial research is of little use unless findings are translated into clinical practice for patient benefit. Our international scoping survey suggests that independent clinical trials that address important questions identified by the dermatology community have substantial, diverse and far reaching impacts on dermatological practice.

\section{What is already known about this topic?}

- Reviews of therapeutic advances in dermatology only comment on possible future practice implications

- We can find no research that attempts to track whether and how clinical trial results actually influences dermatological clinical practice

\section{What does this study add?}

- We scope the way in which four independently funded clinical trials conducted by our team and published over the last 10 years have impacted on clinical practice internationally

- We find that uptake of clinical trial recommendations from large, independent clinical trials is quite high at an international level

- In addition to direct uptake of trial recommendations, we find evidence of indirect uptake such as more confidence in using study drugs for the same disease but in a slightly different way from the trial

- We also find evidence of educational impact of clinical trials on journal clubs and improving study design

- The survey has demonstrated generated interest in the international community on measurement of clinical research impact as an emerging field 


\section{Introduction}

Research impact is a topic that has grown substantially over the last 10 years. Traditionally, research impact was measured by whether an article was published in a prestigious journal with a high "impact factor", or how many times it was cited using citation reports or altmetrics in a given timeframe. ${ }^{1}$ The value of medical research is limited if it is only cited by other researchers in academic reports and not put into any form of action for patient or wider societal benefit. Even if research results are eventually translated into action, the time delay between publishing and implementing clinical findings has been estimated to be around 17 years ${ }^{2}$, which is clearly unacceptable and wasteful.

Greenhalgh et al suggest that impact is achieved when research results confer benefits to wider society in terms of health gain, economy or culture ${ }^{3}$. The measurement of research impact is an emerging field and requires a range of quantitative and qualitative methods such a payback framework, research impact frameworks such as the Canadian Academy of Health Sciences Framework, monetisation models and societal impact assessment. In the UK, research councils determine research excellence of universities by undertaking periodic assessments of research quality and impact which determines how much central funding universities receive. Whereas prestigious publications and infrastructure contributed heavily to determining excellence in older assessments, the 2014 research impact exercise introduced the concept of allowing universities to describe the wider impact of their research outputs ${ }^{4,5}$. During an exercise to evaluate a possible future impact case study on independent clinical trials led by our Centre of Evidence-Based Dermatology (CEBD), it was suggested that the authors conduct a brief international survey of colleagues to evaluate whether the results of those studies were useful or not, and if so in what way. Here, we report the findings of our scoping survey.

\section{Methods}

We undertook a scoping survey, the responses of which were scrutinised for common themes and summarised narratively. We selected the four of our national clinical trials funded independently by Government (National Institute of Health Research) and charity sources (Action Medical Research and Cancer Research UK) that we anticipated would have significant international reach and relevance. The four trials are shown in box 1 and deal with interventions for basal cell carcinoma, cellulitis, bullous pemphigoid and pyoderma gangrenosum. Three of the trials were published in general medical journals and one in a dermatology journal ${ }^{6-9}$. Three of the trials (SWET, BLISTER and STOPGAP) were supported and delivered through the UK Dermatology Clinical Trials Network (UK DCTN) - an independent UK charity dedicated to prioritising and delivering national independent clinical trials in dermatology ${ }^{10}$. All four trials were open access.

We sought to achieve as a high a response as possible by including the request for feedback on our trials all in the body of a single, personalised email, the exact wording of which provided in the supplementary material. The email was kept short and deliberately open in order to avoid responder bias and to elicit themes that might not otherwise have emerged from more targeted questions. The wording was non-directive and included any form of change such as using a given treatment more, using a treatment less or no change. One author ( $\mathrm{HCW}$ ) e-mailed international colleagues from 22 countries spanning five continents. For some countries, we wrote to more than one colleague if we knew they specialised in a particular area (e.g. skin cancer) in order to cover all areas of medical dermatology included in our four exemplar trials. We included responses received from recipients as well as additional responses that they solicited from departmental colleagues or from elsewhere in their country. We did not seek to ascertain collective views through formal routes such as the national dermatological associations.

All responses were tabulated by one author (NKR), themes were identified by HCW and corroborated or added to by others (NKR, JRC and KST), and described narratively. No formal quantitative summaries are provided as they are not appropriate in the context of identifying key themes. Verbatim comments have been checked with senders and permission has been granted in order to comply with data protection rules. 


\section{Results}

In total 33 email requests were sent to international colleagues from 22 countries across five continents and a $100 \%$ response rate was achieved. The full responses in relation to each trial by country are presented in Table 1 in the supplementary material. The following themes were identified:

\section{Assessing clinical trial impact in general}

There was universal interest in the notion of tracking clinical trial impact to ensure clinical benefit. The importance of considering translating articles into other languages such as Chinese was also highlighted, as was the importance of searching beyond national guidelines or other key evidence sources such as UpToDate and patient information sources such as DermNetNZ.

\section{Treatment implications from the studies}

The survey demonstrated increased use of study medications that had shown benefit in the trials.

"This study has changed our practice in Colombia as it has become the first option before starting systemic corticosteroids for all patients (not only for some) in the majority of in-patient dermatology centers."

BLISTER Trial impact, Columbia

In addition, treatments that had been previously used somewhat tentatively were now being used with more confidence as a result of the more robust evidence. There was also a lack of awareness that ciclosporin might be a useful treatment for pyoderma, prior to the STOP GAP Trial.

"I refer to this study a lot because ciclosporin is often forgotten as a good alternative for corticosteroids." STOP GAP Trial impact, The Netherlands

The use of the trial evidence to produce more robust estimates of treatment response that can be incorporated into shared decision making with patients during consultations was also highlighted.

"We cite your study in our decision tool for patients with BCC and your data directly helps patients make more informed decisions." SINS Trial impact, USA

Trial evidence was also used in a modified way. For example the BLISTER study intended to show that a strategy of starting treatment with doxycycline might produce benefit in all severities of pemphigoid, whereas some felt that the data encouraged them to use doxycycline either as a steroid-sparing treatment after treatment with prednisolone had been started or just for milder disease only.

"I prescribe doxycycline frequently for mild disease and have used it as a steroid sparing agent." BLISTER Trial impact, New Zealand

\section{The potential impact of trials was dependent on the external validity of the studies relevant to specific countries.}

Although these were pragmatic trials designed to reflect clinical practice in the UK, several aspects impacted on the applicability of the results to other countries. For example, in many of the surveyed countries, cases of cellulitis were not dealt with by dermatologists, so it is unclear whether the trial findings were taken up by other clinicians using different care pathways in those countries.

There also appeared to be variations in the prevalence of dermatological conditions in some countries conditions such as cellulitis are rarely seen in some countries such as Iran, Japan and Israel, for reasons that are unclear.

Variations also existed in whether study medications were available in some countries e.g. oral penicillin was not available in Taiwan and there was a tendency to use more cephalosporins in Korea due to high rates of penicillin allergy. 
Drug availability aside, there were variations in whether study medications were permitted to be prescribed for the target conditions in some countries e.g. ciclosporin was not permitted for treating pyoderma gangrenosum in several countries due to high cost. In some countries, despite a treatment being available, it were not covered by health insurance, thus limiting the applicability of the results.

\section{Other educational implications}

Beyond the specific trial results, impacts for education and sharing of best practice were highlighted, such as papers being discussed and critically appraised in departmental journal clubs and on-line fora, serving an important educational purpose.

"I have also seen this paper discussed in on-line communities and journal clubs several times in Spain and internationally, and it has been the main guide for my therapeutic decisions since its publication." STOP GAP Trial impact, Spain

Our trials were also useful beyond the results, in that they served as exemplars of good trial design, registration and complete reporting.

"it serves as a rigorous methodological model for conducting high-quality clinical trials for basal cell carcinoma. The international SPIRIT guidelines for trial protocols quote sections of the SINS trial protocol as model examples of how to write a protocol." SINS Trial impact, Canada

The use of videos on trial websites was also highlighted as an effective mean of communicating complex highlevel information in a short time and which can also be used as teaching aids ${ }^{11}$.

"This study was very impactful. I can clearly recall when it was presented in our journal clubs, including your breakthrough role in the video presenting the study!" BLISTER Trial impact, Israel

The mere act of surveying colleagues was also a stimulus to read the original research that they might have missed. This was particularly the case for the pyoderma gangrenosum study that was published in the British Medical Journal.

\section{Discussion}

This brief international scoping survey has revealed new insights into the way clinical trial research may or not impact on dermatological clinical practice around the world. Our scoping survey exceeded our expectations in terms of revealing the diversity of impacts of our four independently-funded clinical trials. In addition to simply documenting whether trial results had directly changed practice, a host of indirect effects such as using drugs already available with more confidence, using the trial results to improve shared decision making and educational benefits such as journal club discussions were identified. Respondents clearly welcomed the concept of reflecting on how evidence had impacted on their clinical practice. The time course of the translation into clinical practice of the four clinical trials (published in 2013, 2015 and two in 2017) is a lot quicker than the average of 17 years previously suggested ${ }^{12}$, but this could be because the trials were published in high impact journals, the study drugs were already available in most countries, and because those surveyed were early evidence adopters. The open-access nature of our four trial publications may have also contributed to the reach of the studies. Open access publication is now being encouraged internationally by the Plan $\mathrm{S}$ coalition, but for those not able to pay for open access, attention to partners/influencers, being clear about your message, and considering a range of channels including videos and social media may help to enhance dissemination ${ }^{14}$.

The survey provide some assurance that the studies conducted by the CEBD and UK DCTN are the sorts of studies that have wide international appeal and clinical significance. Perhaps this is because the trials all addressed key questions that had been identified in previous systematic reviews ${ }^{15-18}$ and prioritised by clinicians and patients through the UK DCTN ${ }^{19}$. Two of the UK DCTN trials in this survey (penicillin for recurrent cellulitis (PATCH) and doxycycline for pemphigoid (BLISTER)) deal with inexpensive medicines that had been 
used for many years by some, which may be beneficial but had never been tested properly. The SINS study was the first independent comparison of topical imiquimod against the reference standard of excisional surgery that provided stable estimates of treatment response - an active comparison study that industry would not usually tackle.

Study strengths include the wide global reach across 22 countries in all five continents and the $100 \%$ response rate. The completeness and frankness of responses to all four trials was also appreciated. We did not publish a study protocol prospectively as we did not set up the survey as a formal research study. The results were so interesting that we felt it appropriate to share them more widely for educational purposes. We have also not attempted to report all of the items as suggested by standards for reporting qualitative research ${ }^{20}$ because most were not appropriate for a simple survey. We did however observe basic principles of analysing qualitative comments through thematic analysis that was corroborated by others. We also report all of the responses verbatim - positive and negative so that readers can come to their own conclusion on what respondents actually said and meant. It might be argued that because all of the respondents were colleagues of one of the authors (HCW) that they were more likely to provide favourable responses. Against this notion, it will be noted that the original email gave permission for respondents to indicate if there was "nothing much to report here" in terms of impact. Indeed, some responses did report that the trials had 'no or little' influence, also suggesting that the answers

Pressures on researchers by Universities to constantly generate research income and to "publish or perish"21 are likely to confer a type of researcher behaviour that assumes that the job is done once a funded study is published, and to move onto the next study instead of engaging with clinicians and service providers, policy makers and patients to implement the results. The late Alessandro Liberati ${ }^{22}$ called this the "the butterfly behaviour of researchers, moving onto the next flower well before the previous one has been fully exploited." Citation in guidelines is an easy way of tracking impact, but counts do not indicate how the study has been cited, and provides no evidence of direct patient benefit. The science of impact assessment and implementation science ${ }^{12,23}$ in dermatology is very much in its infancy. We hope this short report will stimulate others to invest more in translating research findings into practice, and to track the impact of research to ensure that patients benefit. 
Box 1: The four dermatology clinical trials used for tracking impact in this study

1. Williams HC, Bath-Hextall F, Ozolins M, Armstrong SJ, Colver GB, Perkins W, Miller PSJ; Surgery Versus Imiquimod for Nodular and Superficial Basal Cell Carcinoma (SINS) Study Group. Surgery Versus 5\% Imiquimod for Nodular and Superficial Basal Cell Carcinoma: 5-Year Results of the SINS Randomized Controlled Trial. J Invest Dermatol. 2017 Mar;137(3):614-619. https://doi.org/10.1016/j.jid.2016.10.019

Participants randomised: 501 people with nodular or superficial basal cell carcinoma

Interventions compared: excisional surgery (4-mm margin) vs topical imiquimod (5\%, once daily)

Headline result: Although surgery is clearly superior to imiquimod, this study shows sustained benefit for lesions that respond early to topical imiquimod and presents a useful alternative treatment option for people with basal cell carcinoma

Trial website: https://www.nottingham.ac.uk/research/groups/cebd/projects/4non-melanoma/sins.aspx

2. Thomas KS, Crook AM, Nunn AJ, Foster KA, Mason JM, Chalmers JR, Nasr IS, Brindle RJ, English J, Meredith SK, Reynolds NJ, de Berker D, Mortimer PS, Williams HC; U.K. Dermatology Clinical Trials Network's PATCH I Trial Team. Penicillin to prevent recurrent leg cellulitis. N Engl J Med. 2013 May 2;368(18):1695-703. http://dx.doi.org/10.1056/NEJMoa1206300

Participants randomised: 274 people with recurrent cellulitis

Interventions compared: oral phenoxymethylpenicillin (250mg, twice daily) vs oral placebo for 12 months

Headline result: In patients with recurrent cellulitis of the leg, penicillin was effective in preventing subsequent attacks during prophylaxis, but the protective effect diminished progressively once drug therapy was stopped

Trial website: https://www.nottingham.ac.uk/research/groups/cebd/projects/patch.aspx

3. Williams HC, Wojnarowska F, Kirtschig G, Mason J, Godec TR, Schmidt E, Chalmers JR, Childs M, Walton S, Harman K, Chapman A, Whitham D, Nunn AJ; UK Dermatology Clinical Trials Network BLISTER Study Group. Doxycycline versus prednisolone as an initial treatment strategy for bullous pemphigoid: a pragmatic, noninferiority, randomised controlled trial. Lancet. 2017 Apr 22;389(10079):1630-1638.

https://doi.org/10.1016/S0140-6736(17)30560-3

Participants randomised: 253 people with bullous pemphigoid

Interventions compared: oral doxycycline ( $200 \mathrm{mg} /$ day) vs oral prednisolone $(0.5 \mathrm{mg} / \mathrm{kg} /$ day)

Headline result: Starting pemphigoid treatment with doxycycline results in reasonable blister control and much better long term safety (morbidity and mortality) than a strategy of starting with oral prednisolone

Trial website: www.blistertrial.co.uk

4. Ormerod AD, Thomas KS, Craig FE, Mitchell E, Greenlaw N, Norrie J, Mason JM, Walton S, Johnston GA, Williams HC; UK Dermatology Clinical Trials Network's STOP GAP Team. Comparison of the two most commonly used treatments for pyoderma gangrenosum: results of the STOP GAP randomised controlled trial. BMJ. 2015 Jun https://doi.org/10.1136/bmj.h2958 
4. Ormerod AD, Thomas KS, Craig FE, Mitchell E, Greenlaw N, Norrie J, Mason JM, Walton S, Johnston GA, Williams HC; UK Dermatology Clinical Trials Network's STOP GAP Team. Comparison of the two most commonly used treatments for pyoderma gangrenosum: results of the STOP GAP randomised controlled trial. BMJ. 2015 Jun https://doi.org/10.1136/bmj.h2958

Participants randomised: 112 with pyoderma gangrenosum requiring systemic treatment

Interventions compared: Oral prednisolone $(0.75 \mathrm{mg} / \mathrm{kg} /$ day $)$ vs oral ciclosporin $(4 \mathrm{mg} / \mathrm{kg} / \mathrm{day})$

Headline result: These two commonly used treatments for pyoderma gangrenosum only achieve about $50 \%$ healing at 6 months and choice for individual patients is likely to depend on adverse effect profiles of the drugs in relation to patient co-morbidity

Trial website: www.stopgaptrial.co.uk 


\section{Acknowledgements}

We thank Professor Marion Walker from the University of Nottingham who suggested undertaking an international survey.

We also thank all respondents: Katrina Abuabara, Sebastien Barbarot, An-Wen Chan, Ching-Chi Chi, Lei Chu, Mary-Margaret Chren, Robert Dellavalle, Aaron Drucker, Alireza Firooz, Masutaka Furue, Ignacio Garcia-Doval, Urbà González, Kyu Han, Nicole Kelleners-Smeets, Nonhlanhla Khumalo, Laurence Lecleach, Yael Leshem, Eleni Linos, Deedee Murrell, Luigi Naldi, Tamar Nijsten, Amanda Oakley, Saumya Panda, Yuping Ran, Gloria Sanclemente, Marit Saunes, Enno Schmidt, Jochen Schmitt, Hiroshi Shimizu, Michael Sladden, Ake Svensson, Roberto Takaoka, Jacob Thyssen, Hideyuki Ujiie, Carl-Fredrik Wahlgren.

\section{Funding and conflicts}

This study was not funded directly by any funding body. Three of the authors of this article (HW, JC and KT) were also authors on the four trials explored in the survey.

\section{Supplementary material:}

Verbatim responses by country

Copy of actual email sent to colleagues 


\section{References}

Nestor MS, Fischer D, Arnold D, Berman B, Del Rosso JQ. Rethinking the Journal Impact Factor and Publishing in the Digital Age. J Clin Aesthet Dermatol 2020; 13: 12-7.

Morris ZS, Wooding S, Grant J. The answer is 17 years, what is the question: understanding time lags in translational research. J $R$ Soc Med 2011; 104: 510-20.

Greenhalgh T, Raftery J, Hanney S, Glover M. Research impact: a narrative review. BMC Med 2016; 14: 78.

https://www.ref.ac.uk/2014

https://impact.ref.ac.uk/casestudies/Results.aspx?val=Show\%20All.

Thomas KS, Dean T, O'Leary C, Sach TH, Koller K, Frost A, Williams HC. A randomised controlled trial of ion-exchange water softeners for the treatment of eczema in children. PLoS Med 2011; 8: e1000395. Williams HC, Wojnarowska F, Kirtschig G, Mason J, Godec TR, Schmidt E, Chalmers JR, Childs M, Walton S, Harman K, Chapman A, Whitham D, Nunn AJ. Doxycycline versus prednisolone as an initial treatment strategy for bullous pemphigoid: a pragmatic, non-inferiority, randomised controlled trial. Lancet 2017; 389: 1630-8.

Ormerod AD, Thomas KS, Craig FE, Mitchell E, Greenlaw N, Norrie J, Mason JM, Walton S, Johnston GA, Williams HC. Comparison of the two most commonly used treatments for pyoderma gangrenosum: results of the STOP GAP randomised controlled trial. Bmj 2015; 350: h2958. Thomas KS, Crook AM, Nunn AJ, Foster KA, Mason JM, Chalmers JR, Nasr IS, Brindle RJ, English J, Meredith SK, Reynolds NJ, de Berker D, Mortimer PS, Williams HC. Penicillin to prevent recurrent leg cellulitis. N Engl J Med 2013; 368: 1695-703.

www.ukdctn.org/

www.blisterstudy.co.uk

Ashrafzadeh S, Metlay JP, Choudhry NK, Emmons KM, Asgari MM. Using Implementation Science to Optimize the Uptake of Evidence-Based Medicine into Dermatology Practice. J Invest Dermatol 2020; 140: $952-8$.

https://www.coalition-s.org/ https://www.nihr.ac.uk/documents/how-to-disseminate-your-research/19951 Kirtschig G, Middleton P, Bennett C, Murrell DF, Wojnarowska F, Khumalo NP. Interventions for bullous pemphigoid. Cochrane Database Syst Rev 2010; 2010: Cd002292.

Bath-Hextall FJ, Perkins W, Bong J, Williams HC. Interventions for basal cell carcinoma of the skin. Cochrane Database Syst Rev 2007: Cd003412.

Reichrath J, Bens G, Bonowitz A, Tilgen W. Treatment recommendations for pyoderma gangrenosum: an evidence-based review of the literature based on more than 350 patients. J Am Acad Dermatol 2005; 53: 273-83.

Dilemmas when managing cellulitis. Drug Ther Bull 2003; 41: 43-6.

Layfield C, Clarke T, Thomas K, Williams HC. Developing a network in a neglected area of clinical research: the UK Dermatology Clinical Trials Network Clin. Invest. 2011; 1: 943-9.

O'Brien BC, Harris IB, Beckman TJ, Reed DA, Cook DA. Standards for reporting qualitative research: a synthesis of recommendations. Acad Med 2014; 89: 1245-51.

Kwon JH, Denlinger CE. Commentary: Publish or perish at our collective peril. J Thorac Cardiovasc Surg 2020; 160: 735-6.

Magrini N, Smith R. Alessandro Liberati. BMJ 2012; 344: e1101.

Bauer MS, Damschroder L, Hagedorn H, Smith J, Kilbourne AM. An introduction to implementation science for the non-specialist. BMC Psychol 2015; 3: 32. 